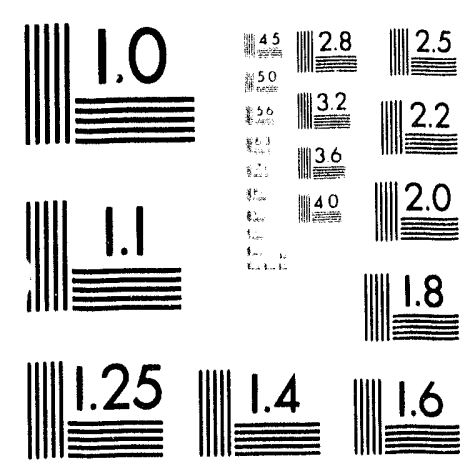



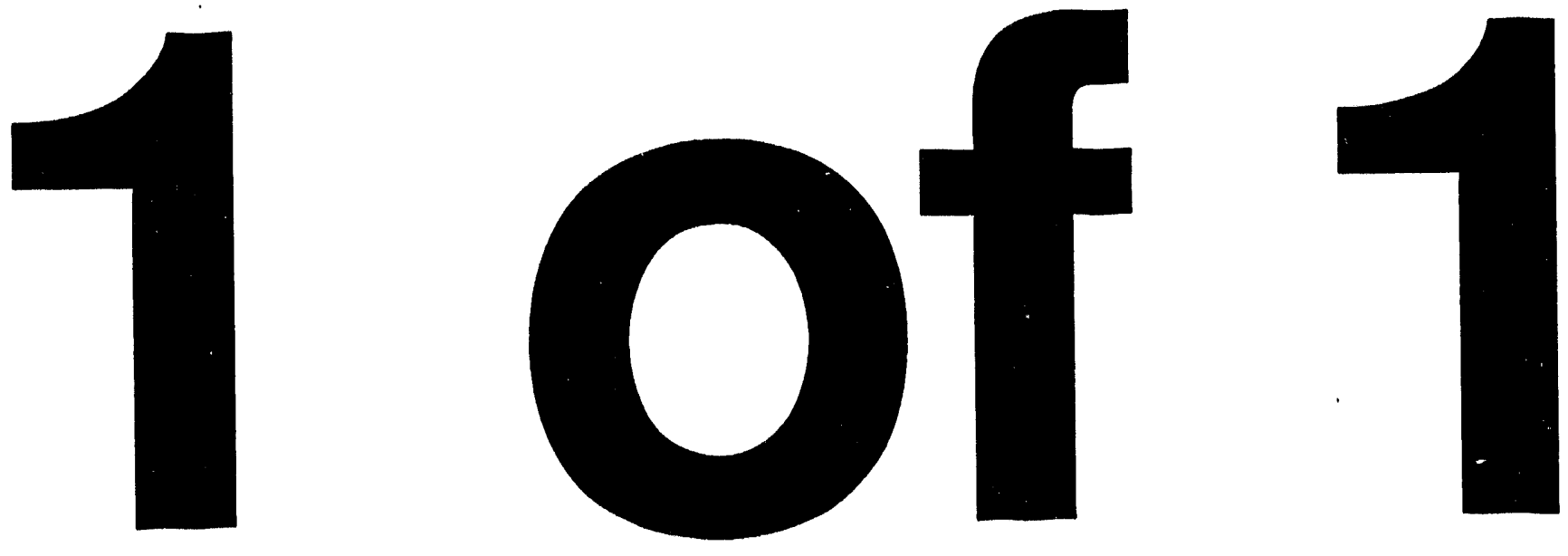


\title{
High Energy Resolution X-ray Spectrometer for High Count Rate XRF Applications
}

\author{
C.S. Rossington, N.W. Madden and K. Chapman \\ Engineering Division \\ Lawrence Berkeley Laboratory \\ University of California, Berkeley CA 94720
}

August 1993

This work was supported by the Director, Office of Energy Research, Office of Biological and Environmental Research, Analytical Technology Division of the U.S. Department of Energy under Contract Number DE-AC03-76SF00098. 


\title{
HIGH ENERGY RESOLUTION X-RAY SPECTROMETER FOR HIGH COUNT RATE XRF APPLICATIONS
}

\author{
C. S. Rossington, N. W. Madden and K. Chapman \\ Lawrence Berkeley Laboratory \\ University of California \\ Berkeley, CA 94720
}

\begin{abstract}
A new $\mathrm{x}$-ray spectrometer has been constructed which incorporates a novel large area, low capacitance $\mathrm{Si}(\mathrm{Li})$ detector and a low noise JFET (junction field effect transistor) preamplifier. The spectrometer operates at high count rates without the conventional compromise in energy resolution. For example, at an amplifier peaking time of $1 \mu \mathrm{sec}$ and a

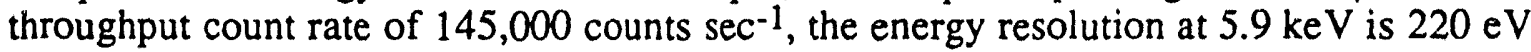
FWHM. Commercially available spectrometers utilizing conventicnal geometry $\mathrm{Si}(\mathrm{Li})$ detectors with areas equivalent to the new detector have resolutions on the order of $540 \mathrm{eV}$ under the same conditions. Conventional $x$-ray spectrometers offering high energy resolution must employ detectors with areas one-tenth the size of the new LBL detector $\left(20 \mathrm{~mm}^{2}\right.$ compared with $200 \mathrm{~mm}^{2}$ ). However, even with the use of the smaller area detectors, the energy resolution of a commercial system is typically limited to approximately $300 \mathrm{eV}$ (again, at $1 \mu \mathrm{sec}$ and $5.9 \mathrm{keV}$ ) due to the noise of the commercially available JFET's. The new large area detector is useful in high count rate applications, but is also useful in the detection of weak photon signals, in which it is desirable to subtend as large an angle of the available photon flux as possible, while still maintaining excellent energy resolution. X-ray fluorescence data from the new spectrometer is shown in comparison to a commercially available system in the analysis of a dilute multi-element material, and also in conjunction with high count rate synchrotron EXAFS applications.
\end{abstract}

\section{INTRODUCTION}

The general problem of detecting high count rate photon fluxes while still maintaining good energy resolution arises in many $x$-ray applications, and particularly so in conjunction with the very high intensity photon fluxes available from $x$-ray synchrotron sources. The $x$ ray spectrometer energy resolution and its ability to handle high count rates are inversely related and at amplifier pulse-processing times of less than $10 \mu \mathrm{sec}$, the series noise can be modeled after a simple CR-RC filter as shown in Equation 1:1 


$$
\Delta E=\frac{\left(C_{d}+C_{f}+C_{s}\right) \varepsilon B 2.35}{q \sqrt{T_{p}}}
$$

where $\Delta \mathrm{E}$ is the electronic component of the energy resolution in $\mathrm{eV}, \mathrm{C}_{\mathrm{d}}$ is the detector capacitance in farads, $\mathrm{C}_{\mathrm{f}}$ is the JFET capacitance, $\mathrm{C}_{\mathrm{S}}$ is any stray capacitance arising from the capacitive coupling of spectrometer components in close proximity to the detector and JFET, $\varepsilon$ is the average energy required to create an electron-hole pair in the detector material in $\mathrm{eV}$ per electron-hole pair, $B$ is the channel noise of the JFET measured in $V / \sqrt{\mathrm{Hz}}, q$ is the electronic charge in Coulombs, and $T_{p}$ is the amplifier peaking time in seconds. It is obvious from the equation above that the energy resolution of an $\mathrm{x}$-ray spectrometer, at short amplifier pulse-processing times of less than approximately $10 \mu \mathrm{sec}$, can be improved by reducing the capacitances of both the detector and the JFET preamplifier. However, reducing the capacitance of the detector has traditionally meant reducing the detector area, which is not desirable for many applications, and the capacitance of commercially available JFET's has been limited to not less than 3-4 pf. We have addressed these issues by constructing an $\mathrm{x}-$ ray spectrometer utilizing a new large area, low capacitance $\mathrm{Si}(\mathrm{Li})$ detector $^{2}$ and a low capacitance Pentafet ${ }^{*}$ JFET preamplifier. ${ }^{3}$ The new spectrometer has been compared to a commercially available low noise system for the $x$-ray fluorescence analysis of a dilute multielement material. The new spectrometer has also been used for two high count rate synchrotron EXAFS applications. The data from these three applications are discussed.
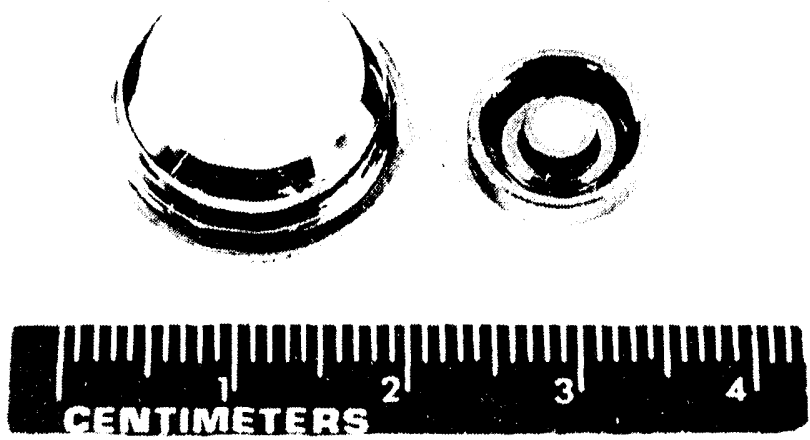

Figure 1. Photograph of the new large area, low capacitance Si(Li) detector compared with a conventional $\mathrm{Si}(\mathrm{Li})$ detector of equivalent capacitance $(0.5 \mathrm{pf})$.

\section{SPECTROMETER}

Figure 1 is a photograph comparing the new large area $\mathrm{Si}(\mathrm{Li})$ detector with a conventional $\mathrm{Si}(\mathrm{Li})$ detector of equivalent capacitance. The capacitance of each of these devices is $0.5 \mathrm{pf}$, while the area of the new detector is $\sim 200 \mathrm{~mm}^{2}$ compared with $\sim 20 \mathrm{~mm}^{2}$ for the conventional structure. The fabrication procedures and detector performance characteristics for the new device have been discussed previously. 2

Figure 2 shows the calculated system electronic noise as a function of amplifier peaking time for the new spectrometer utilizing the new large area detector and low noise JFET, compared with a commercial system utilizing a small area detector of equivalent capacitance and commercially available JFET, and compared to a system employing a conventional large area detector and commercially available JFET. The improvement in energy resolution for the new spectrometer means that the system can be operated at much higher count rates than is

\footnotetext{
* Pentafet is a registered trademark of Link Analytical, High Wycombe, Bucks, England.
} 
typically possible for single element detector spectrometers, without the traditional compromise in energy resolution. For example, as is shown in Figure 3 and Table 1, the new spectrometer can be operated at a maximum throughput rate of 145,000 counts per second ai $1 \mu \mathrm{sec}$ peaking time, while still maintaining a very low electronic noise of $205 \mathrm{eV}$, using triangular amplifier shaping. This count rate is approximately 2.5 times that for a conventional system of equivalent noise, as is shown in the $\mathrm{Fe}^{55}$ spectra of Figure 4. Figure 5 shows the response to $\mathrm{Fe}^{55}$ and the improvement in energy resolution for the new spectrometer compared to a low noise commercial system. Both spectrometers were operating at $1 \mu \mathrm{sec}$ amplifier peaking time, with a resulting $220 \mathrm{eV}$ FWHM at $5.9 \mathrm{keV}$ for the new spectrometer and $\sim 320 \mathrm{eV}$ FWHM for the commercial system.

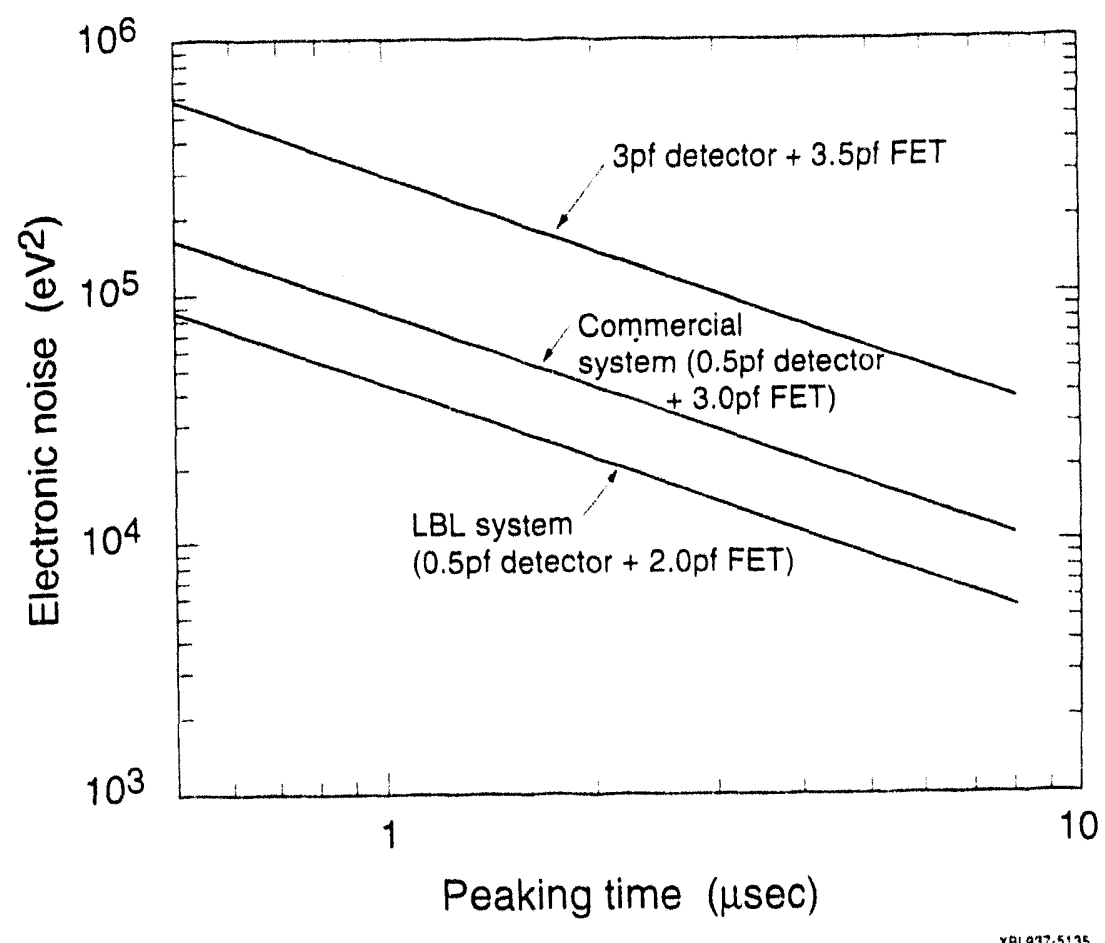

Figure 2. Electronic noise as a function of amplifier peaking time for the new large area detector and low noise JFET $(0.5 \mathrm{pf}$ detector and $2.0 \mathrm{pf}$ JFET), a commercial spectrometer with a small area detector of equivalent capacitance with a commercially available JFET ( $0.5 \mathrm{pf}$ detector and $3.0 \mathrm{pf}$ JFET), and a conventional large area detector with a commercially available JFET ( $3 \mathrm{pf}$ detector and $3.5 \mathrm{pf}$ JFET).

Table 1. Maximum throughput count rates and electronic noise for various short peaking times for three different spectrometers: LBL spectrometer with large area low capacitance detector and low capacitance Pentafet JFET, commercial spectrometer with small area low capacitance detector and commercially available JFET, spectrometer with conventional large area detector and commercially available JFET.

\begin{tabular}{ccccc}
\hline $\begin{array}{c}\text { Peaking } \\
\text { time } \\
(\mu \mathrm{sec})\end{array}$ & $\begin{array}{c}\text { Max. count } \\
\text { rate } \\
\left(\mathrm{sec}^{-1}\right)\end{array}$ & $\begin{array}{c}\mathrm{LBL} \\
200 \mathrm{~mm}^{2}\end{array}$ & $\begin{array}{c}30 \mathrm{~mm}^{2} \\
\text { conventional }\end{array}$ & $\begin{array}{c}200 \mathrm{~mm}^{2} \\
\text { conventional }\end{array}$ \\
\cline { 3 - 5 } \cline { 3 - 5 } & 145,000 & 205 & 290 & 540 \\
1.0 & 110,000 & 180 & 255 & 470 \\
2.3 & 58,000 & 130 & 185 & 340 \\
4.0 & 36,000 & 100 & 145 & 270 \\
\hline
\end{tabular}




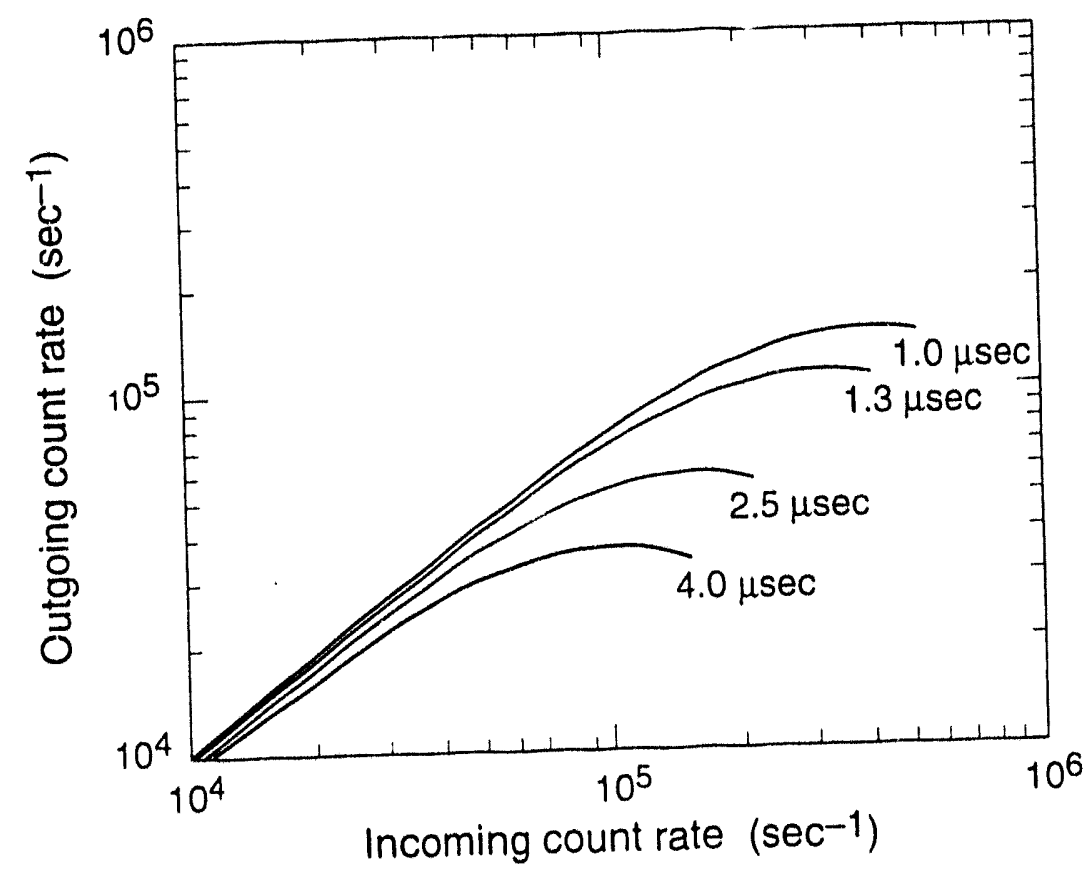

Figure 3. Incoming count rate (ICR) versus outgoing count rate (OCR) for conventional low noise $x$-ray spectrometers as a function of amplifier peaking time. $\left(O C R=I C R\left(\exp -I C R 2.5 T_{p}\right)\right.$

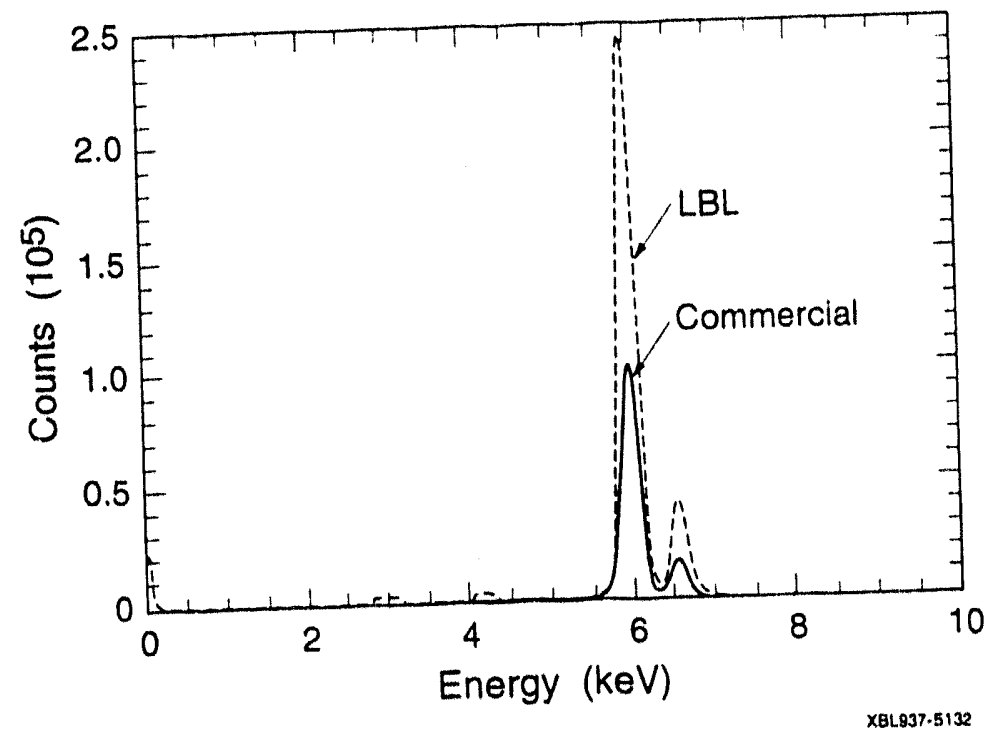

Figure 4. $\mathrm{Fe}^{55}$ spectra for the new spectrometer at $1 \mu \mathrm{sec}$ peaking time and throughput rate of 130,000 counts $\mathrm{sec}^{-1}$, compared with a low noise commercial system at $2.5 \mu \mathrm{sec}$ peaking time and a throughput rate of 50,000 counts $\mathrm{sec}^{-1}$ (FWHM at $5.9 \mathrm{keV} \sim 220 \mathrm{eV}$ for both systems).

\section{APPLICATIONS}

The new spectrometer has been used in three different applications, the first being in the $x$-ray fluorescence analysis of a glass material containing various elements in the $0.5-12$ weight \% range. The sample was fluoresced using an $8 \mathrm{keV}$ rotating anode copper $\mathrm{x}$-ray tube. The fluorescence signal was weak, due to the low concentrations of elements and sample thickness of $0.2 \mathrm{~mm}$. Figure 6 compares the fluorescence $x$-ray spectra for the multi- 
element material using the new spectrometer with the $200 \mathrm{~mm}^{2}$ area detector and a comparable low noise commercial system utilizing a $20 \mathrm{~mm}^{2}$ area Si(Li) detector. The spectra were collected under identical conditions, normalized for live time, and with equivalent energy resolutions of $\sim 220 \mathrm{eV} \mathrm{FWHM}$ at $5.9 \mathrm{keV}$ achieved at $1 \mu \mathrm{sec}$ and $2.5 \mu \mathrm{sec}$ amplifier peaking time for the LBL and commercial systems, respectively. The count rate was 10 times higher for the LBL spectrometer, due to the 10-fold increase in detector area. Again, the increase in detector area was achieved without the traditional compromise in energy resolution. (The count rates from the weak fluorescence signal were far from the maximum possible operating rates, which is why the increase in count rate due to detector area alone is observed).

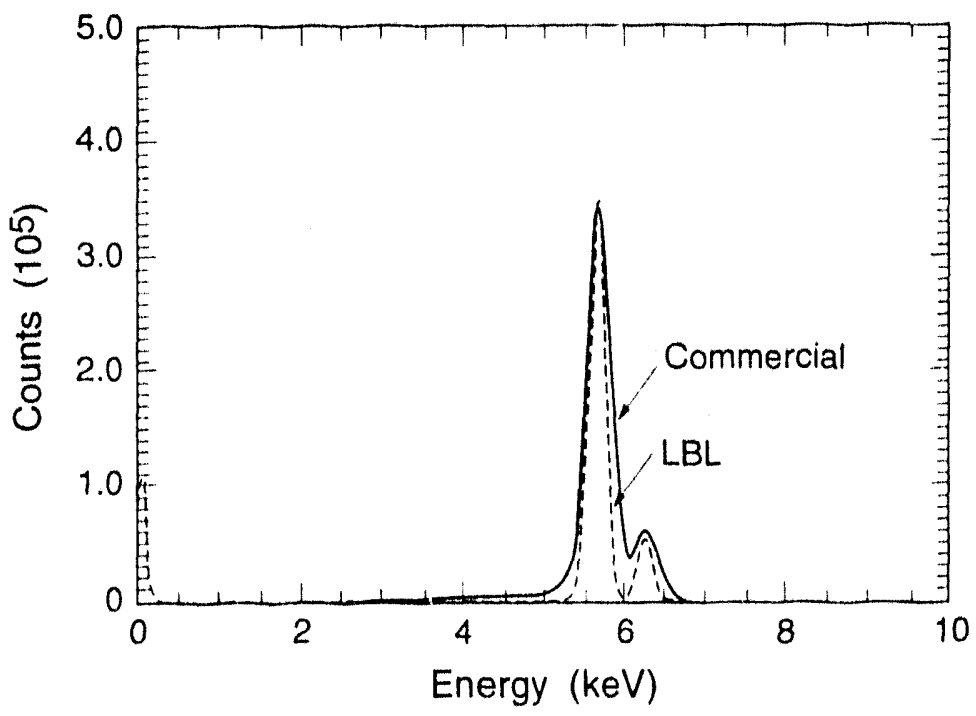

XBL.937.5133

Figure 5. $\mathrm{Fe}^{55}$ spectra for the new spectrometer at $1 \mu \mathrm{sec}$ peaking time (FWHM at $5.9 \mathrm{keV} \sim 220 \mathrm{eV}$ ) compared with a low noise commercial system at $1 \mu \mathrm{sec}$ peaking time (FWHM at $5.9 \mathrm{keV} \sim 320 \mathrm{eV}$ ).

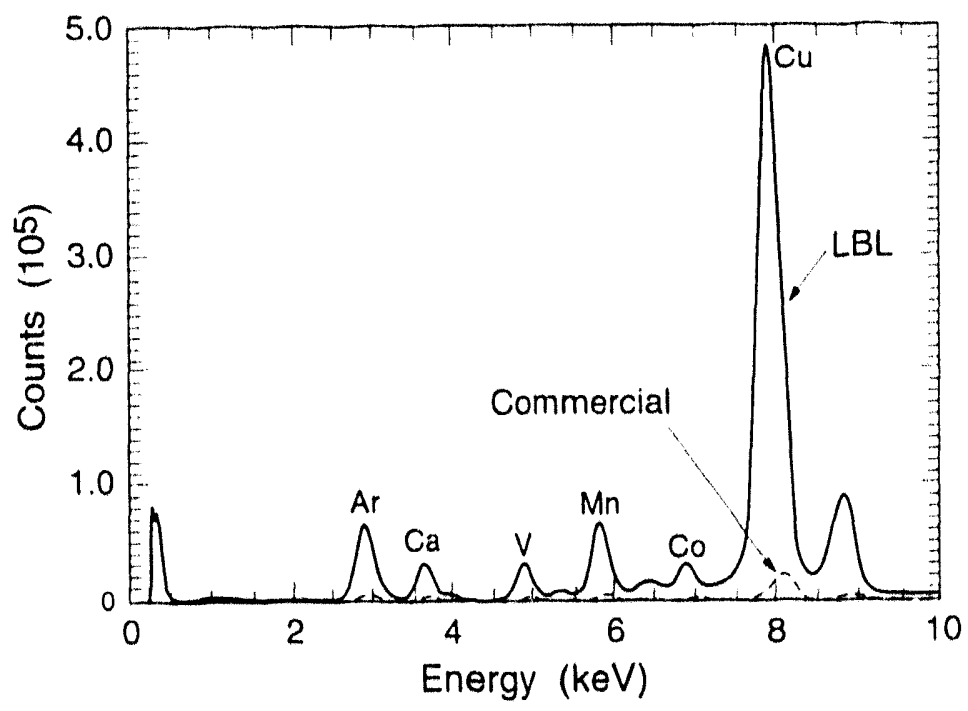

XBL.937.5134

Figure 6. X-ray spectra from a multi-element material fluoresced by an $8 \mathrm{keV}$ copper anode $\mathrm{x}$-ray tube. The count rate from the new spectrometer is 10 times that of the commercial system due to the 10-fold increase in detector area; the energy resolutions of the two systems are equivalent $(-220 \mathrm{eV} \mathrm{FWHM}$ at $8 \mathrm{keV})$, and spectrometer live times have been normalized. 
The spectrometer has also been used in two different EXAFS (extended $x$-ray absorption fine structure spectroscopy) applications. The first was in the detection of $\mathrm{Cl} \mathrm{K- \alpha}$ fluorescence, in which excellent energy resolution was required to separate the $2660 \mathrm{eV} \mathrm{Cl}$ $\mathrm{K}-\alpha$ signal from the synchrotron monochromator scatter peak at $\geq 2820 \mathrm{eV}$. Figure 7 shows the fluorescence spectrum from a $\mathrm{ZnSe}: \mathrm{Cl}$ sample fluoresced with a $2830 \mathrm{eV}$ synchrotron beam. The spectrometei was operating at a $2.5 \mu \mathrm{sec}$ peaking time, a throughput rate of 45,000 counts $\mathrm{sec}^{-1}$ and achieved an energy resolution of $\sim 150 \mathrm{eV} \mathrm{FWHM}$ at $2660 \mathrm{eV}$. The second EXAFS application involved the detection of $U$ L- $\alpha$-rays from an organic compound. This application did not have strict energy resolution requirements as the U L- $\alpha$ peak was well separated in energy from the scatter peak of the synchrotron probe beam. The spectrum of Figure 8 was collected at an amplifier peaking time of $0.5 \mu \mathrm{sec}$ (which was the fastest shaping time of the available amplifier) and a throughput rate of 120,000 counts $\mathrm{sec}^{-1}$, while achieving an energy resolution of $\sim 350 \mathrm{eV}$ FWHM at $13.6 \mathrm{keV}$.

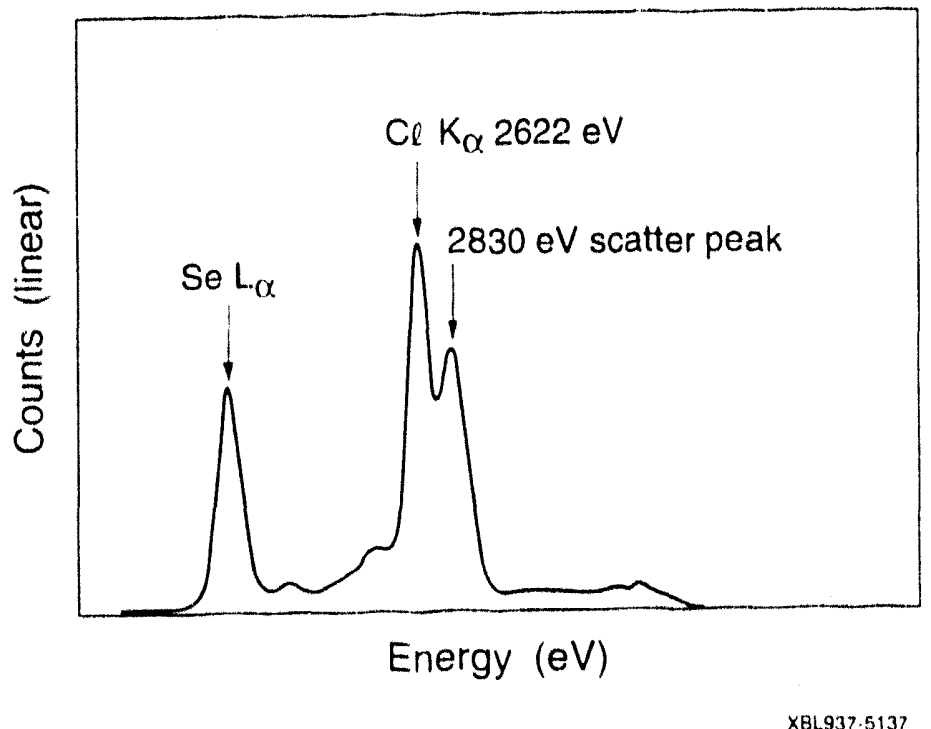

Figure 7. X-ray spectrum from a Cl-doped $\mathrm{ZnSe}$ semiconductor fluoresced by a $2830 \mathrm{eV}$ monochromatic synchrotron source, used for subsequent EXAFS analysis. Spectrometer parameters: $2.5 \mu \mathrm{sec}$ peaking time, 45,000 counts sec-1 throughput rate, $-150 \mathrm{eV}$ FWHM at $2.6 \mathrm{kcV}$.

These EXAFS results can be considered in comparison to a commercially available thirteen-element germanium detector array which many researchers use for EXAFS data collection. The 13-element array, each detector element having a diameter of $10 \mathrm{~mm}$, achieves approximately $180 \mathrm{eV} \mathrm{FWHM}$ at $5.9 \mathrm{keV}$ at an $8 \mu \mathrm{sec}$ amplifier peaking time. At the $8 \mu \mathrm{sec}$ peaking time, the maximum throughput count rate for EXAFS applications is approximately 11,000 counts $\mathrm{sec}^{-1}$ per detector element. The new LBL spectrometer achieves $180 \mathrm{eV} \mathrm{FWHM}$ at $5.9 \mathrm{keV}$ at a $2 \mu \mathrm{sec}$ amplifier peaking time, which results in a throughput count rate of 44,500 counts $\mathrm{sec}^{-1}$. Although the total count rate for the 13element array is 3.25 times greater than for the single element LBL detector, the signal-tobackground is lower, as is explained as follows: In EXAF' experiments using polarized synchrotron $x$-ray beams, the least arnount of scatter signal (scattering of the synchrotron probe beam) occurs at $90^{\circ}$ to the polarized beam. ${ }^{4}$ Thus, the optimum placement of the detector, for the highest signal-to-background, is at $90^{\circ}$ to the beam. Detector elements in an array detector, which fan out from the center of the detector, are at angles less than, or greater than, $90^{\circ}$ and thus the signal-to-background degrades as the distance from the center increases. Calculations and experimental data show that the total signal-to-background for 
the thirteen-element array, for typical EXAFS experimental geometries, is approximately half that for the single element at the center of the array. 5,6 In other words, the single element LBL detector would have one-third the count rate, but twice the signal-to-background, and in addition is much less expensive to construct and much less complicated to operate.

In addition to the three described applications, the new large area detector would also be useful in x-ray astrophysics applications, in which the $\mathrm{x}$-ray signal is relatively weak and the count rate could be improved 10 -fold over conventional high energy resolution detectors due to the 10-fold increase in detector area. The new detector could also improve the sensitivity of TXRF (total reflection X-ray fluorescence) experiments, again by offering an increase in detector area compared with the conventional detectors now being used.?

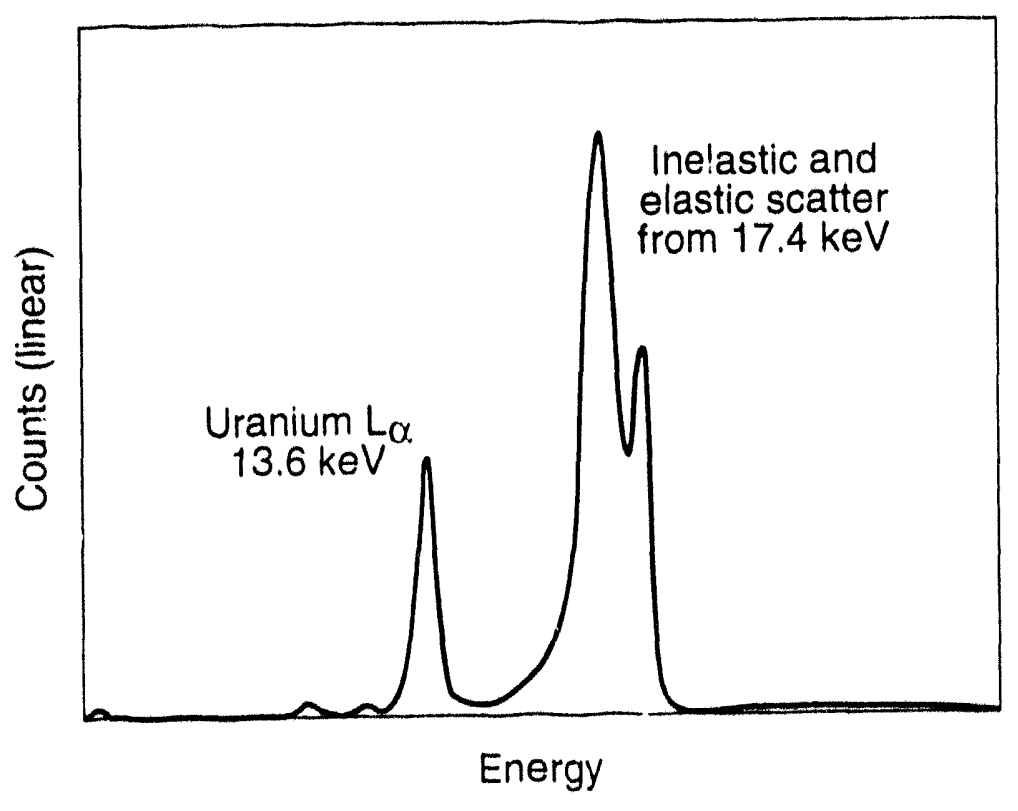

Figure 8. X-ray spectrum from an organic compound containing uranium, nuoresced by a $17.4 \mathrm{keV}$ monochromatic synchrotron source, used for subsequent EXAFS analysis. Spectrometer parameters: $0.5 \mu \mathrm{sec}$

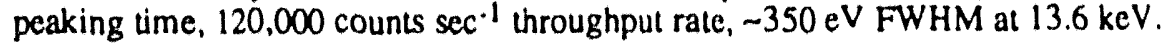

\section{ACKNOWLEDGMENTS}

The authors wish to thank D. Malone and C. P. Cork for assistance in the construction and testing of the spectrometer, K. M. Yu for the chlorine EXAFS data and J. Bucher for the uranium EXAFS data. We would also like to thank T. Nashashibi of Link Analytical for the generous gift of their "Pentafet" FET. This work was supported by the Director, Office of Energy Research, Office of Biological and Environmental Research, Analytical Technology Division, of the U.S. Department of Energy under Contract No. DE-AC03-76SF00098. Reference to a company or product name does not imply approval or recommendation of the product by the University of Califormia or the U.S. Department of Energy to the exclusion of others that may be suitable. 


\section{REFERENCES}

1. F. S. Goulding and D. A. Landis, Semiconductor detector spectrometer electronics, in: "Nuclear Spectroscopy and Reactions, Part A," J. Cerny, ed., Academic Press, New York (1974).

2. C. S. Rossington, P. M. Fine and N. W. Madden, Large area, low capacitance Si(Li) detectors for high rate $x$-ray applications, LBL report $\# 32243$, accepted for publication in IEEE Trans. Nucl. Sci.

3. T. Nashashibi and G. White, A low noise FET with integrated charge restoration for radiation detectors, IEEE Trans. Nucl. Sci. 37(2):452 (1990).

4. A. L. Hanson, The polarization of $\mathrm{x}$-rays scattered into $90^{\circ}, \mathrm{Nucl}$. Instr. and Meth. A249:515 (1986).

5. K. Janssens, Monte Carlo simulation of synchrotron radiation induced $x$-ray fluorescence spectra: a useful tool for spectrometer design and calibration, presented at the Denver X-ray Conference, Denver, CO, Aug. 2-6, 1993.

6. V. de Rose, private communication.

7. P. Wobrauschek, private communication. 

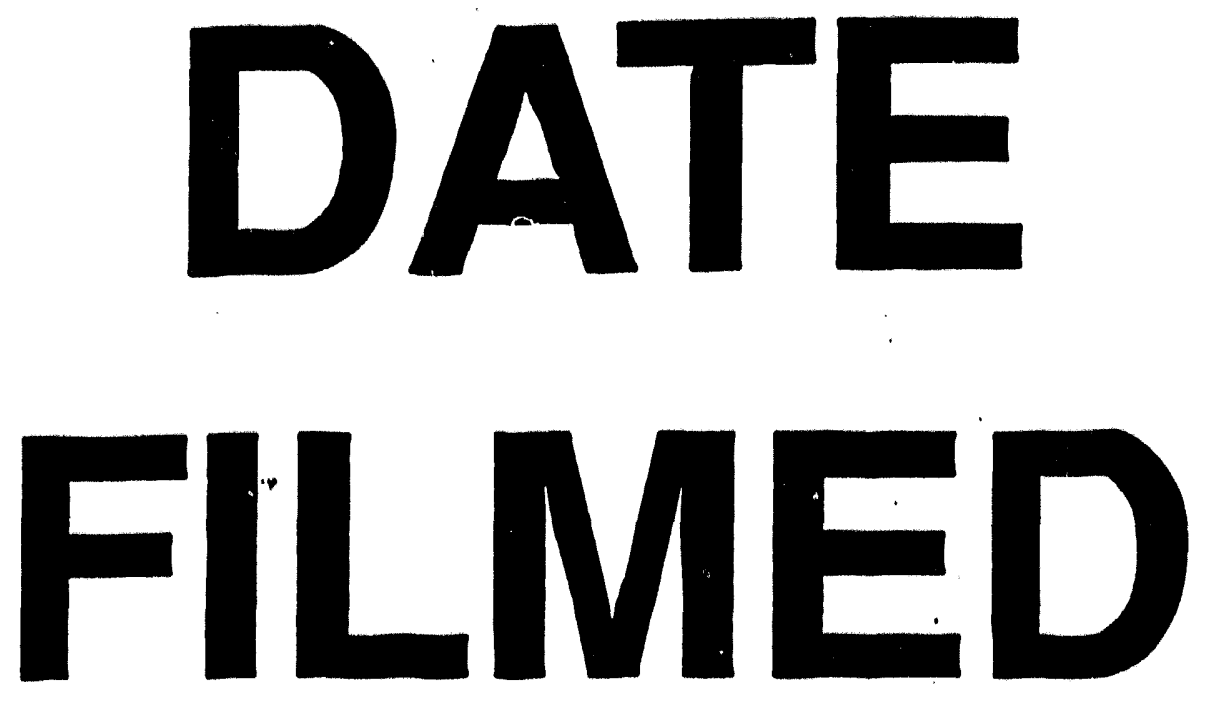

$12 / 14 / 93$
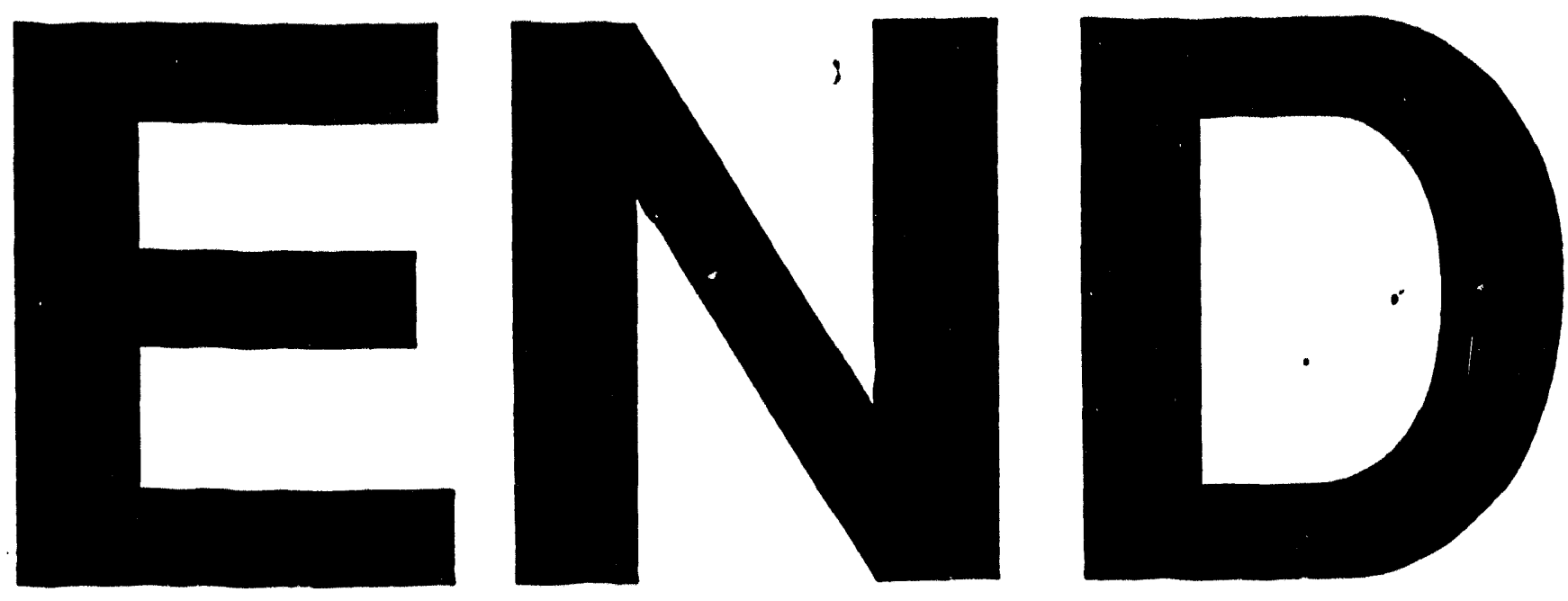
$$
--{ }_{-1}
$$ 